\title{
Animal Model Analysis based on the Characteristics of Clinical Symptoms of Alcoholic Hepatic Injury
}

\author{
Le KANG \\ Henan University of Traditional Chinese Medicine \\ Zhengzhou, Henan, 450046, China \\ e-mail: akangle056@126.com
}

Xiaoyan FANG

Henan University of Traditional Chinese Medicine Zhengzhou, Henan, 450046, China, e-mail: fxylele@yeah.net

\author{
Zhenya HU \\ Henan University of Traditional Chinese Medicine \\ Zhengzhou, Henan, 450046, China \\ e-mail: 769519542@qq.com \\ Mingsan MIAO \\ Henan University of Traditional Chinese Medicine \\ Zhengzhou, Henan, 450046, China \\ e-mail: miaomingsan@163.com
}

\begin{abstract}
Objective: Based on the analysis of the characteristics of traditional Chinese medicine and Western medicine clinical symptoms of alcoholic liver injury. Method: In this paper, the characteristics and modeling methods of alcoholic liver injury animal model were summarized through analyzing the clinical diagnostic criteria and therapeutic effect criteria of alcoholic liver injury in Western and Chinese medicine. By comparing the clinical symptoms and the standard, the current animal model and clinical symptoms of the anastomosis were analyzed and discussed. Result: The existing animal model of alcoholic liver injury showed that the similarity of alcoholic liver injury was higher and the clinical symptoms were more similar. Conclusion: The model of alcoholic liver injury remains to be improved, It should be further based on the clinical symptoms and indicators of patients to improve, to provide a more reliable basis for clinical.
\end{abstract}

Keywords-alcoholic liver injury; clinical disease characteristics; animal model

\section{INTRODUCTION}

Alcoholic hepatic injury is a liver disease caused by long-term heavy drinking. In the early stage, it is usually expressed as fatty liver, which can be developed into alcoholic hepatitis, liver fibrosis and cirrhosis. The main clinical features are nausea, vomiting, jaundice, liver enlargement and tenderness. And can be complicated with liver function failure and upper gastrointestinal bleeding, etc. Severe alcoholism can induce a wide range of liver cell necrosis, and even liver failure. Alcoholic liver disease is one of the common liver diseases in our country, which seriously endangers the people's health. Therefore, it is of important guiding significance to study the pathogenesis, pathological and physiological changes, prevention and treatment of liver injury, and to study the mechanism of liver injury, and to develop a reliable animal model of alcoholic liver injury.

\section{ETIOlOgY AND Pathogenesis of Alcoholic LIVER INJURY}

\section{A. Traditional Chinese Medicine Etiology and Pathogenesis}

Traditional Chinese medicine has not been mentioned in the literature and alcoholic liver injury similar to the exact name. But according to the specific etiology, pathogenesis and clinical manifestations of alcoholic liver injury, alcoholic liver injury can be classified as alcoholism, "Alcohol addiction", "hypochondriac pain" and other diseases. The view of Chinese medicine is that the wine is hot and toxic of the goods, and the Qi is radical into the heart, liver, lung, stomach. Over the drink will hurt the liver and gallbladder spleen and stomach. "" Compendium of Materia Medica, "said:" Less alcohol can reconcile Qi and blood, more alcohol damage the body. "" Treatments on the theory "said" alcoholic is toxic and hot, if drinking too much will seep zang-fu organs and meridian, and then it will produce a variety of diseases. A large number of alcohol can lead to endogenous heat, block the liver and gallbladder qi, spleen and stomach obstruction. In addition, gas, blood, sputum and wet each other knot, loss of liver and kidney yin, causing liver and kidney deficiency [1]. The internal cause of alcoholic liver injury is due to lack of ferrite endowment. The external cause is due to over-drinking and excessive drinking, which leads to spleen and stomach injury, damp-heat internal resistance, spleen loss endocrine, Qi and blood from the hypochondriac pain, blood stasis Results in

\section{B. Modern Medical Etiology and Pathogenesis}

It is generally believed that oxidative stress, inflammation, immunity, mitochondrial dysfunction, apoptosis and acetaldehyde toxicity are closely related to the pathogenesis of alcoholic liver injury, and it is equivalent to the individual differences, gender differences and alcohol dehydrogenase and acetate dehydrogenase gene polymorphism in the body of alcohol into acetaldehyde in the belly of the heart beat from alcohol. 
medical thought, and acetaldehyde as a highly toxic compound can promote lipid peroxidation, resulting in damage to the mitochondria, reduced glutathione, and increased collagen synthesis . Oxidative stress is considered to be one of the most important pathogenesis of alcoholic liver injury and plays an important role in the formation and development of alcoholic liver injury. Acetaldehyde and malondialdehyde (MDA), a lipid peroxidation product, can synergize with protein to form MDA, acetaldehyde and protein hybridization adducts. It not only directly causes cell damage, but also as antigenic stimuli, stimulating the body to produce the appropriate antibodies, and causing immune injury[2]. Malondialdehyde is the final product of lipid peroxidation, and it can act on nuclear factor NF-kB, and the results of the release of inflammatory factor gene expression increased; MDA can also make the cell membrane fluidity and permeability disorders, leading to liver cells Structural and functional damage, the formation of the body's free radical damage. Superoxide dismutase (SOD), an important antioxidant in the liver, is the only superoxide anion scavenger in vivo and plays a key role in the pathogenesis of alcoholic liver injury and plays an important role in the recovery of liver injury. Its main function is to convert the superoxide anion into oxygen, and then through the role of hydrogen peroxide into water and reduced to non-toxic. Tumor necrosis factor alpha (TNF-) is an important pro-inflammatory factor and immune regulatory factor, and it is also a key factor in the occurrence and development of alcoholic fatty liver .Excessive intake of alcohol often secondary to endotoxin, and endotoxin can stimulate intrahepatic Kupffer cells release TNF- $\alpha$, with the liver cell membrane TNF-areceptor binding, increase intracellular reactive oxygen species formation, and promote superoxide Oxygen anion generated from the mitochondrial complex, resulting in increased intrahepatic oxide and antioxidant reduction, resulting in oxidative stress and peroxidation of membrane phospholipids, causing intrahepatic pain and depletion of adenosine triphosphate and glutathione levels, Lipid accumulation, liver cell dysfunction, induced hepatocyte necrosis and apoptosis.

\section{Clinical Treatment of Alcoholic Liver INJURY}

\section{A. Treatment of Alcoholic Liver Injury with Traditional Chinese Medicine}

Generally, dispersing stagnated liver qi for relieving qi stagnation, regulating qi-flowing for strengthening spleen, promoting blood circulation for removing blood stasis, heat-clearing, damp-inhibiting, nourish liver and kidney and Chinese medicine detoxification is the main drug use rules of liver. Use higher frequency of 10 kinds of Chinese herbal medicine is Radix bupleuri, Citrus trifoliata, Pueraria, Flos puerariae, Astragalus membranaceus, Salvia miltiorrhiza, Rhizoma alismatis, Poria cocos, Rhizoma Atractylodis Macrocephalae, Hawthorn[1].

\section{B. Western Medicine in the Treatment of Alcoholic Liver Injury}

At present, the principle of the treatment of alcoholic liver disease in modern medicine is still symptomatic treatment, stop drinking and nutritional support to reduce the severity of alcoholic liver disease. To improve the existing secondary malnutrition and symptomatic treatment of alcoholic cirrhosis and its complications, Western medicine treatment is: (1)Antioxidant: as zinc, selenium, vitamins. (2) Glucocorticoid. (3)Drugs of fight and improve ethanol metabolism: Metadoxine, Adenosine methionine. (4)Medicine for protecting liver cells and promoting regeneration of liver cells: glycyrrhizic acid preparation, silymarin, Polyene phosphatidylcholine, reduced glutathione, etc[3].

\section{ANAlysis OF THE Clinical CHARACTERISTICS OF ALCOHOLIC LIVER INJURY}

\section{A. Diagnostic Criteria of Traditional Chinese Medicine}

Su Wen, Fu Zhong, Lun Pian said: "Huang Di said: there is a confidant of the fullness of the disease, the morning to eat at night to eat, what disease is this? Qibo answered: a meteorism." Jinkui Yaolue · Huang Dan Bing Mai Zheng Bing Zhi Di Shi Wu said: "Heart trouble Nong, Not eat, then want to vomit, called alcoholic jaundice." Shengji Zonglu, volume seventy-third, bibulosity said: "Summarize the stomach weak, because drinking too much, while the wine is hot. Thirst and lead to drink. In case of airway obstruction. Wine and drink are unchanged. Stop at the side of the flank. Gathering them, Its shape can be shaped by hand, or when there is a sound. The sharp feeling of fullness in chest and hypochondrium, causing pain or nausea, muscle skinny can not eat, only drink can alleviate after drinking. So he has a drinking habit." Yifang Xuanyao, Juan Zhi Qi, Wu Dan Men said: "Have jaundice disease, hot and humid hot in the spleen, the yellow face limb is correct. The jaundice has five names: one is called jaundice ...... four alcoholic jaundice, body and eyes are yellow, annoyance pain, yellow urine, red spots appearing on the face, which are due to drinking in the famine, drunk when wind into the water ...... . These five aspects of the symptoms are not the same, but the sector has shown a yellow color, the root causes are caused by damp heat." Zhengyin Maizhi-Juan Si.Xie Wu Lun.Fu Wu Jing Xie Wan.Jiu Ji Wu Jing Xie Wan said: "Every dawn, stomach pain, pain after shitting, pull out is yellow foam, urine red. Some like the gruel. This is the symptom of the alcoholic dyspepsia." Explain what the alcoholic dyspepsia: "The man of excessive drinking, or drinking cool wine, his stomach hurted, humid and hot environment, accumulated into heat, fire Yin Mao, is the dawn diarrhea."

Based on the summary of the above, the majority of Chinese researchers to determine the clinical alcoholic liver injury is: (1)Hypochondriac pain, abdominal pain and distention. (2)Jaundice. (3)Weak,poor appetite,dry. (4)Epistaxis,bleeding from the gum. (5)Hematemesis. (6) Hematochezia. (7) If serious will faint. (8)There will be tongue petechia, dark purple tongue, and its color is yellow or white, pulse string sunken etc[4]. 


\section{B. Diagnostic Criteria of Western Medicine}

Clinical diagnostic criteria of alcoholic liver disease: The Chinese Medical Association of Hepatology and alcoholic liver disease study group of the February 2006 amendments to the guidelines for management of alcoholic liver disease, the diagnostic criteria of alcoholic liver disease: (1)There is a long history of drinking,usually more than 5 years, converted into ethanol amount,the amount of male consumption of more than $40 \mathrm{~g} / \mathrm{d}$, and the women is more than $20 \mathrm{~g} / \mathrm{d}$, or a large number of drinking history within 2 weeks, equivalent to the amount of ethanol is greater than $80 \mathrm{~g} /$ days. (2) The clinical symptoms were nonspecific,asymptomatic or have abdominal pain, loss of appetite, fatigue, weight loss,jaundice. (3)Serum AST, ALT, Y-GT, total bilirubin, prothrombin time and average hematocrit (MCV) were elevated[5], hyaluronic acid (HA) and endothelin (ET) index increased. Lipid peroxidation indexes such as malondialdehyde (MDA) increased, superoxide dismutase (SOD) reduced glutathione peroxidase (GSH-PX) decreased with different degrees of TG or higher. Reduce the indexes of energy metabolism activity can drink these indexes returned to normal within 4 weeks usually. AST/ALT>2, help todiagnose[6,7]. (4)Liver ultrasound or CT examination showed, fatty degeneration of liver cells, CT determination of liver volume increased, visible diffuse and localized fatty liver parenchyma image; ultrasonography of liver injury, Ye Zengda and caudate, pseudo parallel tube syndrome. (5)To exclude the infection, drug and toxic liver injury.

In accordance with the article (1)(2)(3) and (5)or (1)(2) (4) and (5)can diagnose the ALD; Only in line with (1)(2) and (5) can be diagnosed the ALD.

\section{The Characteristics AND Detection INDEXES OF THE ANIMAL MODEL OF ALCOHOLIC LIVER INJURY AND THE ANALYSIS OF THE DEGREE OF AgREEMENT With THE CliniCAL DiagNostic INDEXES OF TRADITIONAL CHINESE MEDICINE AND WESTERN MEDICINE.}

Types of alcoholic liver injury model in general: Rats and mice, rabbits, dogs, pigs, snow leopard, primate animal etc[8], and the animal experiment is used to rats and mice as the main animal. The main modeling methods are the following, and please see the table I.

TABLE 1 ANimal Model AND Model ANALysis of COMMON ALCOHOLIC LiVER INJURY

Symptom and its agreement with the clinical symptoms of Chinese and Western Medicine

Symptoms: Mild walking instability, stiff action, serious drunk lethargy,even shortness of breath;most of the animal listlessness, weight loss, loose stools diarrhea,drowsiness, hair dull and erect, normal recovery of about $1 \sim 2 \mathrm{~h}$. Eating less than normal animals. Fatty degeneration of liver cells with mild balloon like change and inflammatory cell infiltration.

56 degrees liquor Indicators: ALT, AST, TG, TCH, MDA, GSH-PX, SOD.

filling the Features: The method is cumbersome, the amount of alcohol is difficult to ensure that every time, and easy to cause the stomach[9,10]. complications of gastric intubation. A small number of animals have frequent vomiting, can not tolerate alcohol and death of gastric perfusion.

According to the clinical diagnostic criteria: Chinese Medicine: (1)(3)(4)(5)(6)(7). Goodness of fit: 75.5\%. Western medicine: (1)(2)(3)(5) Goodness of fit: $72.5 \%$.

Symptoms: Mild walking instability, stiff action;serious drunk lethargy, even shortness of breath, normal recovery of about 30 60

Intraperitoneal injection of a certain concentration of ethanol[11] minutes. Eating less than normal animals. Fatty degeneration of liver cells with mild balloon like change and inflammatory cell infiltration.

Indicators: ALT, AST, TG, TCH, MDA, GSH-PX, SOD, GSH

Features: doesn't accord with human normal drinking habits, and the accuracy of the model is poor.

According to the clinical diagnostic criteria: Chinese Medicine: (1)(3)(7). Goodness of fit: $42.9 \%$. Western medicine: (1)(2)(3)(4)Goodness of fit: $72 \%$.

Symptoms: Light walking, slow, or drunk sleepiness, about $1 \mathrm{~h}$ began to recover. Different degrees of liver damage, fatty degeneration of liver cells, central necrosis of the liver, infiltration of inflammatory cells, activation of complement activation, platelet function and microcirculation disturbance in the liver.

Index: ALT, AST, GGT, body weight, liver weight.

Tsukamato-French Features: The model of high repetition rate can be studied in vivo metabolism mechanism of alcohol is very good, but the production of the model requires the placement of gastric tube operation is difficult and expensive, so it is difficult to popularize. In addition, because the model is directly injected into the stomach containing ethanol liquid feed, ethanol content is not high, it is difficult to produce alcoholic liver cirrhosis.

According to the diagnostic criteria of TCM: (1)(3)(4)(7). Goodness of fit: 57.1\%. Western medicine : (1)(2)(3)(4)(5)Goodness of fit: $62 \%$.

Symptoms: hepatic steatosis, ballooning degeneration and necrosis and mononuclear cell infiltration, platelet hyperfunction and microcirculation.

Lieber-Decarli liquid Indicators: AST, ALT, SOD, MDA, GSH, NOS, iNOS

food[13]

Features: Easy to operate, easy to copy, almost no mortality. High rate of formation, good stability, can be copied alcoholic fatty liver and hepatitis. But the long period of model making, pathological changes is limited to the mild fat, it is difficult to form characteristic liver fibrosis.

According to the diagnostic criteria of TCM: (3)(4)Goodness of fit: the 28.6\%. Western medicine: (1)(2)(3)(5)Goodness of fit: 69\%. 
TABle 1 Animal Model and Model Analysis of Common Alcoholic Liver Injury(Cont.)

\begin{tabular}{ll}
\hline Animal model & Symptom and its agreement with the clinical symptoms of Chinese and Western Medicine \\
\hline & Symptoms: Fatty degeneration of liver cells and neutrophils infiltration of inflammatory cells and fibrosis around the central vein of \\
Drinking water to join alcoholic liver damage. & \\
alcohol, and with low & Indicators: ALT, AST, TG, TCH, MDA, SOD \\
fat, high protein and & Features: Simple, diet composition ratio and human proximity. But it is difficult to assess and control the role of nutritional factors \\
containing a certain & associated with alcohol consumption in the occurrence of alcoholic liver disease. It is difficult to control the intake of alcohol, and \\
amount of choline and the liver of rats is strong in alcohol metabolism, easy to form tolerance to alcohol, it is difficult to maintain a high blood alcohol \\
sugar[12] \\
concentration. Compared with other alcoholic liver disease models, this model has a mild degree of steatosis.
\end{tabular}

According to the degree of agreement with traditional Chinese medicine and Western medicine clinical symptoms with high degree of classification. High degree of agreement: Western medicine $>70 \%$, Chinese medicine $>70 \%$. General matchness: Western medicine $>60 \%$, Chinese medicine $>60 \%$. Low degree of conformity: Western medicine $<50 \%$, Chinese medicine $<50 \%$.

\section{DISCUSSION}

Alcohol is an important cause of human liver injury, animal models must be established to study the pathogenesis of alcoholic liver injury in order to seek prevention and treatment measures. Domestic scholars to use alcohol gavage or drinking water in the method of adding alcohol, with a high degree of clinical fit[13]. Although the latter reduced rat mortality, but difficult to maintain effective blood alcohol concentration. Alcoholic liver injury model is difficult to establish. Intraperitoneal injection of a certain concentration of ethanol does not meet the normal human drinking, and the application has some limitations. Lieber-Decarl model, Tsukamoto-French model is based on a certain percentage of total calorie intake to determine the amount of alcohol consumption, does not meet the normal alcohol intake, while the Tsukamoto-French model requires some special techniques and equipment. But Lieber-Decarli liquid food supplementation model and the physiological situation does not match. Therefore, alcoholic liver injury model of alcoholic rats was stable, pathological changes and clinical similar to clinical alcoholic liver disease research to provide a convenient model for the recommendation.

Alcoholic liver damage in human formation of a long time, and the human and animal metabolism of alcohol is different, the healing response to liver damage is also different. Because there are a variety of animal models, because of its predisposing factors, different pathological changes and fatty liver, liver fibrosis formation mechanism is different, the ideal animal model is: Similar to the human disease characteristics. Lesions have a certain development process, high success rate, low mortality, good reproducibility. Modeling method is simple and easy, slow down the disease after modeling to stop reversing, facilitate the study of drug intervention. Therefore, the researchers choose appropriate animal models according to the research purpose, technical proficiency and laboratory conditions, and as far as possible to improve the model, in order to find the ideal model. With the development of science, the use of genetic engineering or molecular biological methods to produce more ideal animal model of alcoholic liver injury, which requires the majority of scholars to actively explore and research.

\section{ACKNOWLEDGEMENTS}

The research work was supported by Transformation project of Henan Province (142201610011), Excellent science and technology innovation team in Henan Province(TCJ2014-391).

\section{REFERENCE}

[1] Liu Yufei, Pan Junying, Lin Lin, et al. Study on the characteristics of traditional Chinese medicine in the treatment of alcoholic liver injury $[\mathrm{J}]$. Information on Traditional Chinese Medicine, 2016,33(3):126-128.

[2] WANG Jun-ming, MIAO Ming-san, QU Ling-bo et al. Status and Proposals of Applying Proteomicsto Research Essence of Liver Injury[J].Chinese Journal of Experimental Traditional Medical Formulae. 2013,19(9):358-361.

[3] ZHAN Zongying, SUN Mingyu. Traditional Chinese Medicine Treatment and Research Progress of Alcoholic Liver Disease[J]. Journal of Sichuan of Traditional Chinese Medicine, 2014,32(10): 182-185.

[4] Clinical analysis of 50 cases of alcoholic liver disease treated with traditional Chinese Medicine[J].CHINA'S NATUROPATHY,2012,20(6):63-64.

[5] Choi JS, Yoon TJ, Kang KR. Glycoprotein isolated from Acanthopanax senti-cosus proteits against hepalotoxicity induced by acute and chronic alcohol treatment[J]. Biol Pharm Bull, 2006, 29: 306-314.

[6] LIU Fang-ping, MA Xin, ZHAO Chang-weiStudy on GSTA1, an early indicator of alcohol induced acute liver injury in mice[J].Chinese Journal of Veterinary Medicine, 2015,51(7):88-86.

[7] ZHU Qiang, WANG Ke, QIAN Xiao-feng,et al.Modelling the chronic alcoholic liver disease in mouse[J].Chinese Journal of Clinicians (Electronic Edition), 2010,4(9):52-55.

[8] WANG Ling1, SUNWu-yi1, JIANG Ling,et al. Advances in animalmodel of alcoholic liver disease[J]. Anhui Medical and Pharmaceutical Journal, 2010,14(7):745-747.

[9] Peng Bo, Miao Mingsan, Wang Yingfang, et al. Effect of Ganlan jiejiudecoction on pathological liver injury rat acute alcoholic liver[J]. Chin J Tradit Chin Med Pharm, 2004,19(8):469.

[10] Song Z, Deaciuc I, Song M. Silymarin protects against acute ethanol-induced hepatotoxicity in mice[J]. Alcohol Clin Exv Res,2006,30:407-413.

[11] Bergheim I, Eagon PK, Dooley S, et al. Alcoholic liver disease and exacerbation by malnutrition and infections: what animal models are currently available?[J]. Ann N Y Acad Sci,2011,1216:41-49.

[12] Guo Yuedong, Ma Lijie. Research progress of animal model of alcoholic liver injury[J]. Inner Mongolia Med J, 2016,48(3):304-305.

Tao Lun. Research progress of experimental animal model of alcoholic liver injury[J]. Medical Innovation of China,2011,8(5):187-188. 